\title{
PERCEPÇÃO DE PAIS/CUIDADOR DE INDIVÍDUOS COM FENILCETONÚRIA, COM E SEM AUTISMO, ACOMPANHADOS EM SERVIÇO DE REFERÊNCIA EM TRIAGEM NEONATAL
}

\author{
Isabella Regina Gomes de Queiroz*, Milena Pereira Pondé** \\ Autora correspondente: Milena Pereira Pondé. Email: isabellaqueiroz@bahiana.edu.br \\ * Associação de Pais e Amigos dos Excepcionais (APAE) de Salvador. \\ ** Escola Bahiana de Medicina e Saúde Pública.
}

\section{Resumo}

Introdução: Fenilcetonúria (FCN) e outros erros de metabolismo estão associados ao autismo. Diagnóstico e tratamento precoces previnem os sintomas. A doença crônica incidindo em etapa precoce interfere na estruturação psíquica e nas aquisições instrumentais do bebê, nos planos real e imaginário. Objetivo: Compreender a percepção de pais/cuidador de indivíduos com fenilcetonúria, com e sem autismo, acompanhados em Serviço de Referência em Triagem Neonatal (SRTN / BA). Métodos: estudo qualitativo exploratório e documental. Foram estudados prontuários de quatro duplas de irmãos com FCN, cada uma composta por um paciente com e outro sem autismo. Nos prontuários, destacou-se relatos sobre: percepção materna, paterna e de irmã cuidadora (de uma das duplas), sobre esses pacientes. A partir da análise de conteúdo, utilizando o programa NVIVO, categorizou-se o material selecionado, agrupando-o, subsequentemente, em árvores temáticas. Nesse artigo serão abordadas as árvores 1) Busca diagnóstica; 2) Funcionamento familiar, focando nos pacientes com autismo. Resultados: Três dos pacientes com autismo nasceram antes do Programa Nacional de Triagem Neonatal (PNTN). Um paciente com autismo e seu irmão realizaram Triagem Neonatal em outro estado, na rede privada, tendo resultado falso negativo. Verificou-se sofrimento e perda do filho idealizado para as mães. Constatou-se diferentes fenótipos e mesma mutação genética em uma das duplas; adoecimento psíquico e desarticulação familiar agravaram a sintomatologia dos pacientes, contribuindo para as diferenças. Considerações finais: Alterações das taxas de fenilalanina e tipo de mutação não esgotam as alterações do funcionamento psíquico dos pacientes - a intervenção interdisciplinar, considerando a estruturação psíquica dos sujeitos, deve ser preconizada.

Palavras-chave: Fenilcetonúrias; Transtorno autístico; Psicologia; Triagem neonatal. 


\begin{abstract}
Introduction: Phenylketonuria (PKU) as other errors of metabolism are associated to autism. Early diagnosis and treatment prevent the symptoms. Chronic disease occurring at a precocious stage affects the psychic structure and the baby instrumental acquisitions, in real and imaginary plans. Objective: To understand the percetption pf parents/caregiver of individuals with PKU, with and without autism, throughout the psychological accompaniment, in a Newborn Screening Reference Center (SRTN/BA). Methods: exploratory and documental qualitative study. The records of four pairs of siblings with PKU were studied, each one composed by a patient with autism and without autism. In the medical records examined, stands out the reports about: maternal perception, parental and caregiver sister (from one of doubles) on these patients. From the content analysis using NVivo software, we categorized up the selected material, grouping it subsequently into thematic trees. In this paper we will discuss the trees 1) Diagnostic Search; 2) Family functioning, focusing on patients with autism. Results: Three of the patients with autism were born before the National Neonatal Screening Program (PNTN). One patient with autism and his brother held Newborn Screening in another state, in private medical health, with false negative result. Suffering and loss of the idealized son for mothers were ratify. Different phenotypes and the same genetic mutation in one of the pairs were found; mental illness and family dislocation aggravated the symptoms of patients contributing to differences. Final Thoughts: Changes in FAL and the type of mutation do not exhaust the changes on the psychic functioning of patients; interdisciplinary intervention, considering the psychic structure of the subject should be recommended in such cases.
\end{abstract}

Keywords: Phenylketonuria; Autistic disorder; Psychology; Newborn Screening.

A relação entre $\mathrm{FCN}$ clássica com diagnóstico tardio e o autismo está bem estabelecida na literatura, ${ }^{(1-6)}$ sendo descrita uma proporção duas vezes maior de autistas nesses casos que na população em geral. ${ }^{(7)}$

A FNC caracteriza-se pela deficiência ou ausência da enzima fenilalanina-hidroxilase (FAH) que converte a fenilalanina (FAL) em tirosina. Decorre de mutações no gene do cromossomo 12q22-q24. Constitui-se em distúrbio genético de herança autossômica recessiva, com mais de 500 mutações identificadas. ${ }^{(3)} \mathrm{Em}$ 2007, foi descrita a relação da mutação IVSiont11g/a (IVSiont546), em homozigose, com o autismo - achados em três casos estudados. ${ }^{(4)}$ Quando diagnóstico e tratamento (através de dieta pobre em proteína) não acontecem precocemente, verifica-se sintomatologias como deficiência intelectual (DI), ${ }^{(8)}$ irritabilidade, agres- sividade, tremores, convulsões, déficit de atenção (DA) e, raramente, autismo. ${ }^{(3)} \mathrm{A}$ triagem para FCN favorece a suspeição clínica desses sintomas em irmãos, parentes e conhecidos de pacientes acompanhados nos serviços de referência em triagem neonatal (SRTN), como possíveis casos de FCN (quando a etiologia é desconhecida) - deve-se, então, convocá-los a diagnóstico diferencial para FCN e ao tratamento subsequentemente. ${ }^{(9)}$

A Triagem Neonatal (TN), através de exame bioquímico, é indicada para diagnóstico precoce da FNC. Deve ser realizada, idealmente, entre o terceiro e o sétimo dia de vida, (3) período pertencente a um tempo em que laço mãe / filho está sendo estabelecido.

A Portaria GM/MS n. ${ }^{\circ} 822 / \mathrm{GM}$ instituiu o Programa Nacional de Triagem Neonatal (PNTN), 
em 6 de junho de 2001, preconizando: testagem para todos recém-nascidos vivos, implantação dos SRTN e acompanhamento multidisciplinar para os casos triados. ${ }^{(10)}$

A FNC é pouco frequente, sendo a proporção encontrada no Brasil de 1:10.000 e na Bahia, 1:16.334 ${ }^{(9)}$ - fator que, possivelmente, corrobora para o desconhecimento médico dessa aminoacidopatia. Sua sintomatologia pode, inclusive, conduzir a equívocos diagnósticos. Dificuldade dos serviços garantirem cobertura de $100 \%$ da triagem para os nascidos/vivos; diagnósticos falsos negativos (por coleta antes do tempo preconizado pelo PNTN - 48 horas); perdas pela busca ativa e, ain$\mathrm{da}$, casos nascidos antes do PNTN apontam para existência de casos sem diagnóstico e sem tratamento, vivendo com distintas sintomatologias da FNC. (9) No Brasil, estima-se que existam cerca de 2 milhões autistas; ${ }^{(11)}$ desses, alguns podem constituir-se em casos de FNC não tratados.

Mães de crianças com anomalias congênitas, tanto quanto mães de crianças com autismo, tendem, inicialmente, a fixarem-se nos aspectos positivos do desenvolvimento dos filhos, negligenciando possíveis alterações nesse processo, como recurso para protegerem a si e a suas crianças de frustrações. ${ }^{(12)} A$ interpretação dos pais para os problemas de desenvolvimento dos filhos como pequenos atrasos, abre uma brecha de esperança, sustentando uma aposta nesse ser, ainda em constituição psíquica. Contudo, a negação do problema prorroga o enfrentamento dos cuidados necessários. É a partir da possibilidade de fazerem o luto do bebê idealizado, que pai e mãe podem se deparar com o bebê real, permitindo o reposicionamento da criança no imaginário dos pais, possibilitando-os que se apropriarem de obstáculos e possibilidades do fiIho. Se a distância entre bebê ideal e real for grande, dificuldades na construção do laço pais/filho podem surgir, ${ }^{(13-15)}$ ocasionando problemas para a constituição psíquica do bebê e para suas aquisições instrumentais. ${ }^{(14)}$

Irritabilidade e alteração do tônus do bebê, presentes nos casos de FNC desde um tempo preco- ce, ${ }^{(3)}$ afetam a dinâmica do diálogo tônico, ${ }^{(16,17)}$ regularmente, presente nas trocas mãe/filho, acarretando dificuldades do agente materno ler os sinais emitidos pelo bebê. ${ }^{(17)} \mathrm{Com}$ a persistência do não tratamento, os primeiros sinais do autismo podem ser manifestos. ${ }^{(3)}$ Bebê com sinais precoces de autismo apresentam alterações no circuito pulsional, ${ }^{(18)}$ favorecendo o desinvestimento materno na criança e na relação. Depressão, dificuldade para conciliar o sono, crises de choro, entre outras são possíveis reações da mãe diante do nascimento do filho com deficiência. ${ }^{(19)}$

A constituição do sujeito é um percurso longo e complexo em que está em jogo um sistema de trocas na relação com um Outro que Ihe constitua e com um terceiro. Uma doença grave pode interferir na estruturação psíquica do bebê ao tornar-se o traço prevalente através do qual a criança é reconhecida pelos seus pais (a partir dos ditos advindos da equipe de saúde). Desenvolvimento e estruturação psíquica, ainda que não possuam atributo de equivalência, representam, por tudo isso, duas ordens que são coincidentes, podendo um incidir sobre o outro. ${ }^{(15)}$

A interdisciplinaridade é preconizada em ações preventivas, dada a complexidade inerente a essa prática. ${ }^{(20,21)}$

A reflexão sobre efeitos na relação pais/filhos, desde a notícia da FNC, é importante para melhor compreensão das repercussões no desenvolvimento, na estruturação psíquica e no tratamento dos pacientes, sobretudo nos casos que cursam com autismo, por serem casos complexos e mais graves. Essa questão faz-se importante pois a FNC, possui tratamento específico, a partir de dietoterapia com baixo aporte proteico e fórmula isenta de FAL, diferentemente de muitos casos de autismo. Casos de diagnóstico de FNC tardios são passíveis de tratamento e, ainda que os danos cerebrais sejam descritos como irreversíveis, observa-se melhora significativa dos pacientes. Objetiva-se compreender a percepção de pais/cuidador de indivíduos com fenilcetonúria, com e sem autismo, acompanhados em um SRTN. 
Trata-se de estudo qualitativo(22) exploratório e documental, cuja fonte de dados constituiu de prontuários de atendimento psicológico de $4 \mathrm{du}$ plas de irmãos com diagnóstico de fenilcetonúria, atendidos em um serviço de referência na cidade de Salvador / BA, que conta com equipe multidisciplinar. Utilizou-se materiais ainda não elaborados para pesquisa, atendendo-se aos requisitos da pesquisa documental. ${ }^{(23,24)}$ Os dados foram levantados de maneira retrospectiva, constituindo-se em estudo qualitativo retrospectivo. Foram incluídas todas as duplas de irmãos com FNC, acompanhadas no SRTN, entre os anos de 2001 e 2014 atendendo aos critérios: serem irmãos germanos, um deles ter sintomas e/ou diagnóstico de autismo, terem sido atendidos no serviço de referência em questão, assinarem o TCLE. Levantou-se dos prontuários informações sobre: percepção materna, paterna e de cuidador a respeito dos indivíduos. Utilizou-se o modelo de análise de conteúdo temático, ${ }^{(22)}$ buscando a detecção de núcleos de sentidos que compõem uma comunicação. Optou-se pelo uso de pseudônimos na identificação dos pacientes, acrescentando o sobrenome Azul àqueles com autismo (cor que representa o dia mundial de conscientização do autismo). Essa pesquisa foi aprovada pelo Comitê de Ética e Pesquisa da Escola Bahiana de Medicina e Saúde Pública; parecer número 518.464 .

A partir dos conteúdos das narrativas dos pais, das mães e da cuidadora dos pacientes, identificou-se as categorias: percurso diagnóstico, impacto do diagnóstico de fenilcetonúria, tratamento da fenilcetonúria, relação familiar (mãe do paciente, pai do paciente), dimensão psíquica inicial do paciente e dimensão psíquica em entrevistas ulteriores. Essas categorias foram agrupadas em quatro árvores temáticas:(22) 1) Busca diagnóstica; 2) Funcionamento familiar (percepção sobre a relação fa- miliar, percepção sobre a mãe do paciente, percepção sobre o pai do paciente); 3) Percepção de pais e/ou cuidador sobre o tratamento da fenilcetonúria; 4) Dimensão psíquica (impacto do diagnóstico de fenilcetonúria, percepção sobre a dimensão psíquica inicial do paciente, percepção sobre a dimensão psíquica em entrevistas subsequentes).

Neste artigo foram abordadas as categorias: 1) Busca diagnóstica; 2) Funcionamento familiar (percepção sobre: relação familiar, mãe do paciente, pai do paciente).

As características apresentadas foram: dupla 1: Alice Azul e Valdemar, TN com resultado falso negativo. Na primeira entrevista no SRTN, manifestaram sintomas distintos e alteração de FAL. Pai professor universitário e mãe artesã (desempregada). Família com bom padrão socioeconômico. Dupla 2: Emília e Antonino Azul. Triados pelo projeto Genética no Sertão, ${ }^{(25)}$ manifestações clínicas distintas, mutação genética $\mathrm{R} 252 \mathrm{~W}^{(26)}$ e alteração de FAL. Antonino Azul realizou tratamento à distância em parceria com rede de saúde do local. Pai lavrador e mãe dona de casa. Pai e pacientes recebem aposentadoria. Emília é tutelada pela irmã cuidadora (destaca-se a importância da sustentação desta em uma função maternante). Dupla 3: pacientes transferidos e com erro de tratamento. Adilson Azul foi diagnosticado para FNC após a TN do irmão menor, Reginaldo, ter positivado. Ambos com alteração nos níveis de FAL no sangue, receberam orientações nutricionais errôneas no SRTN de origem. Pai trabalha com serviços gerais e mãe dona de casa. Dupla 4: pacientes triados e tratados no SRNT do estudo. Aderbal Azul foi diagnosticado após TN da irmã menor (Lindinalva, assintomática) ter positivado. Pai pedreiro e mãe dona de casa. Os dados dos pacientes estão descritos no Quadro 1. 
Quadro 1 - Perfil dos pacientes estudados

\begin{tabular}{|c|c|c|c|c|c|}
\hline & $\begin{array}{c}\text { SitUAÇÃO CLÍNICA } \\
\text { NA la CONSULTA NO } \\
\text { SRTN/BA }\end{array}$ & $\begin{array}{c}\text { DATA DE } \\
\text { NASCIMENTO }\end{array}$ & $\begin{array}{c}\text { IDADE } \\
\text { INÍCIO DE } \\
\text { TRATAMENTO }\end{array}$ & $\begin{array}{l}\text { NíVEIS DE FAL } \\
\text { NA PRIMEIRA } \\
\text { CONSULTA NO } \\
\text { SRTN/BA }\end{array}$ & $\begin{array}{l}\text { IDADE Ia } \\
\text { CONSULTA COM } \\
\text { PSICÓLOGO } \\
\text { SRTN/ BA }\end{array}$ \\
\hline Alice AZUL & $\begin{array}{c}\text { Hiperatividade/ } \\
\text { autismo. }\end{array}$ & 16/11/2000 & $10 \mathrm{a}, 4 \mathrm{~m}$ & $17,23 \mathrm{mg} / \mathrm{dl}$ & $10 \mathrm{a}, 4 \mathrm{~m}$ \\
\hline VALDEMAR & $\begin{array}{c}\text { Falta de atenção/ } \\
\text { agitação. }\end{array}$ & 03/01/2008 & $3 a, 3 m$ e $15 d$ & $20,17 \mathrm{mg} / \mathrm{dl}$ & $3 a, 3 m$ e $21 d$ \\
\hline EMÍLIA & $\begin{array}{l}\text { Deficiência } \\
\text { intelectual. }\end{array}$ & O2/O8/1973 & $35 a, 5 m, 29 d$ & $42,51 \mathrm{mg} / \mathrm{dl}$ & $35 a, 5 m, 29 d$ \\
\hline $\begin{array}{c}\text { ANTONINO } \\
\text { AZUL }\end{array}$ & $\begin{array}{c}\text { Agitação/ } \\
\text { agressividade/ } \\
\text { quadro de autismo. }\end{array}$ & 14/08/1988 & $19 a, 5 m, 18 d$ & Sem registro & $19 a, 5 m, 18 d$ \\
\hline $\begin{array}{l}\text { ADILSON } \\
\text { AzUL }\end{array}$ & $\begin{array}{c}\text { Sintomas de } \\
\text { autismo/agitação. }\end{array}$ & 03/08/2000 & 8 anos & $23,85 \mathrm{mg} / \mathrm{dl}$ & $12 \mathrm{a}, 8 \mathrm{~m}, 12 \mathrm{~d}$ \\
\hline REGINALDO & $\begin{array}{l}\text { Criança típica, } \\
\text { diagnóstico } \\
\text { e tratamento } \\
\text { precoces/agitação. }\end{array}$ & $21 / 08 / 2008$ & $\operatorname{lm}$ e meio & $22,46 \mathrm{mg} / \mathrm{dl}$ & $4 a, 7 m$ e 25 \\
\hline $\begin{array}{l}\text { ADERBAL } \\
\text { AzUL }\end{array}$ & $\begin{array}{c}\text { Quadro de } \\
\text { autismo/ agitação/ } \\
\text { hipotonia grave. }\end{array}$ & 26/O5/1997 & $4 a, 3 m, 4 d$ & Sem registro & $4 a, 11 \mathrm{~m}$ \\
\hline LINDINALVA & $\begin{array}{l}\text { Criança típica, } \\
\text { diagnóstico } \\
\text { e tratamento } \\
\text { precoces. }\end{array}$ & 24/03/2001 & $\operatorname{lm}$ e $9 d$ & Sem registro & $9 \mathrm{~m}$ \\
\hline
\end{tabular}

Fonte: Dados do estudo, cedidos pelo SRTN/ não publicados.

\section{BUSCA DIAGNÓSTICA}

Nessa categoria, foram identificadas variações nos percursos, a depender da história vinculada ao processo de diagnóstico da FNC: a) Não foi feita triagem; b) Resultado falso negativo na triagem neonatal.

Dentre os seis pacientes com diagnóstico tardio, quatro não tiveram suspeição clínica de fenilcetonúria - dois deles com autismo. À exceção de Valdemar, esses pacientes sofreram equívocos diagnósticos ao longo de suas vidas, independente do quadro ser correlato ao autismo ou não.
Quatro pacientes (três com autismo e um com DI) não realizaram a TN - nasceram antes da implantação do PNTN. Emília (DI) e Aderbal Azul apresentaram a mutação, R252W em homozigose. (27) Apesar de haver ingerido alimentos com fenilalanina por maior período de tempo que seu irmão Aderbal Azul (15 anos mais novo), Emília, apresentou sintomas mesmo graves que os dele.

Dois dos pacientes com autismo só tiveram diagnóstico definitivo a partir do resultado positivo da TN dos irmãos.

Adilson Azul teve suspeita de FNC aos 2 anos de idade; foi encaminhado para tratamento em SP, 
o que não se concretizou. O diagnóstico definitivo veio com o resultado positivo da $\mathrm{TN}$, do irmão.

Através do Reginaldo que ele começou a entrar em tratamento[...] já tinha 8 anos, já teve sequela. Caminhou com 4 anos. Procurei tratamento por um ano, dois anos. Aí, disseram que eu fosse pra SP. [...] Eu nem dei ouvido porque eu não tinha condições. (Mãe 3 sobre Adilson Azul).

Sem tratamento, os sintomas foram agravados.

Dois pacientes que tiveram resultado falso negativo para FNC nasceram em hospital da rede privada, em um estado brasileiro onde a coleta de sangue para a realização da TN acontece na maternidade, antes das 48 horas (infringindo o período preconizado pelo PNTN). A hipótese de FNC de Alice Azul deu-se aos 10 anos de idade, após peregrinação por profissionais. Valdemar, apesar dos sintomas (irritabilidade/agitação), nunca despertou atenção de médicos, professores e familiares. A hipótese de FNC, e subsequente solicitação de exame, aconteceu no SRTN, graças à sua agitação, durante a consulta da irmã - o resultado foi positivado. A mãe narra a peregrinação vivida com a filha por distintos profissionais:

Muitos tiques, traços autísticos; sacudir as mãos (em SP). [...] outro neuro [...] hiperativa (em SP, também) [...] Inquieta, não descobriu a fonte. [...] psicólogos falavam que era a educação em casa [...] estava precisando de limite, não me ajudava em nada. [...] Faz acompanhamento psicopedagógico. [...] (Mãer sobre Alice Azul).

Percebe-se que profissionais, dificilmente, relacionam as manifestações clínicas à $F N C^{(9)}$ e que o intercâmbio entre os profissionais que acompanharam o caso não se deu, independentemente da condição econômica familiar.

\section{FUNCIONAMENTO FAMILIAR}

As dinâmicas familiares apresentaram fragilidade das funções exercidas por seus membros; os filhos, em posição vulnerável e de grande sofrimento subjetivo, estiveram inseridos em contexto de desorganização psíquica, com dificuldades no vínculo entre as figuras parentais. Essa categoria está subdividida em: 2.1 Percepção sobre a mãe; 2.2 Percepção sobre o pai.

\section{PERCEPÇÃO SOBRE A MÃE}

Verificou-se sofrimento psíquico materno diante da doença dos filhos; quebra da idealização do filho (mesmo quando o diagnóstico foi precoce), acompanhada de sentimento de desamparo e impotência; adoecimento mental da mãe; compromisso com o tratamento dos filhos; alteração do laço mãe / filho e reposicionamento materno.

Todas apesentaram sofrimento psíquico: "estou angustiada; choro pra caramba." (Mãe de Alice Azul e Valdemar). "[...] muito nervosa e diante da dificuldade dos filhos, chora muito." (Registro psicólogo sobre mãe 4).

O sofrimento foi, também, revelado no sentimento de desamparo e impotência na narrativa de duas mães: '[...] Não tem ninguém da família pra orientar[...] (Mãe sobre dificuldades de observar sinais de atraso de Aderbal Azul). '[...] eu era sozinha para tudo [...]' (Mãe 3 sobre relação com Adilson Azul e Reginaldo).

As mães queixaram-se de exaustão: '[...] É muito para mim [...]' (Relato Mãe de Alice Azul e Valdemar)." Mostraram-se mal colocadas na função materna.

Verificou-se sentimento de impotência a partir do diagnóstico da FNC no caso dos filhos percebidos como saudáveis: "Eu já sabia que não era fácil com o que eu tinha, mas 'vim' outro com a mesma coisa" (Mãe sobre Reginaldo). "Os dois doentes, é muito para mim [...]" (Mãe 1 sobre Valdemar).

Sinais de adoecimento psíquico foram evidentes em três mães:

[...] mãe deprimiu e vive a maior parte do tempo deitada. (Irmã cuidadora sobre relação mãe / Antonino Azul). [...] diz que já havia pensado em se matar. (Mãe de Adilson Azul e Reginaldo). [...] pensa em se matar... uma vez, pensou em tocar fogo nas cortinas da casa. (Mãe de Aderbal Azul e Lindinalva). 
A situação de desencontro perdurou com filhos autistas privados de linguagem: "Penso que não vou conseguir com o Adilson Azul, não sei, ele não para, ele não presta atenção." (Mãe sobre Adilson Azul). Observou-se fechamento no circuito mãe / filho: "Mãe não gosta quando ninguém fala com ele. [...]" (Irmã cuidadora sobre a mãe de Antonino Azul).

Ruptura das trocas mãe $x$ filho, nos casos dos pacientes privados de linguagem agravaram os sintomas: [...] Antes eu não falava com ele. (Mãe 3 sobre Adilson Azul). [...]Vivia em cárcere privado. (Registro psicológico sobre Antonino Azul).

Três das mães relataram dificuldades com a dieta por razões diferentes: desconhecimento sobre alimentos proibidos; dificuldade de acesso a alimentos permitidos; orientações errôneas quanto à dietoterapia em outro SRTN.

\section{PERCEPÇÃO SOBRE O PAI.}

Os pais apresentaram dificuldade na relação com os filhos: ou demonstraram agressividade, ou não participaram suficientemente da vida deles.

Dois pais demonstraram irritabilidade e violência. [...] não é de palavras. Se ele estiver nervoso, ele vai, grita qualquer palavrão com as crianças presentes. "[...] uma vez pegou o cachorro da filha e jogou contra parede" (Mãe sobre pai 1) - a ação de violência foi declarada com o filho autista. O outro pai é narrado como violento em relação ao autista e aos demais filhos: [...] é violento com ele, quando bebe, chuta, não tem paciência; manda afastar quando chega junto. É muito agressivo com os meninos quando bebe [...] se os meninos mexerem com ele... (Mãe sobre pai 4).

A interação era precária nos casos do pai de Emília e Antonino Azul e do pai de Adilson Azul e Reginaldo.

Os pai 1 e 2 nunca compareceram ao serviço.

O comparecimento dos pais 2,3 e 4 na vida dos filhos, quando ocorreu, possibilitou mudanças na relação mãe $\mathrm{x}$ filho, mãe $\mathrm{x}$ pai $\mathrm{x}$ filho: [...]o pai foi botando limite e eu fui atrás". (Mãe sobre pai 3, após entrevista psicológica).
Somente o pai de Lindinalva e Aderbal Azul envolveu-se com a dietoterapia dos filhos.

A partir dos resultados descritos, verificou-se confusão diagnóstica graças ao desconhecimento da fenilcetonúria. Os pacientes, apesar da peregrinação por profissionais de saúde, não obtiveram diagnóstico adequado fora dos serviços especializados.

O silenciamento das mães em relação aos filhos autistas privados de linguagem e a ruptura no sistema de trocas mãe $x$ filho, perceptível nesses casos, estão bem estabelecidos na literatura.

Todas as mães apresentaram manifestações de crises de choro e/ou depressão, ${ }^{(19)}$ conforme descritas na literatura como reação possível mediante nascimento do filho com alguma deficiência. Destacou-se, ainda, ideação suicida em duas delas.

Observou-se na dinâmica pai $x$ filho, mãe $x$ pai $x$ filho uma iminente ruptura com os fundamentos da vida, diante da incomensurável manifestação da pulsão de morte: houve destituição do lugar das crianças em seu atributo de outro, de semelhante e, mesmo, da condição de filhos. A falência da "Lei do Pai" apontou para fragilização da vida familiar; a carência do pacto das leis da cultura instaurou-se, apresentando-se como efeito da desestruturação dos vínculos. ${ }^{(27)}$

Apesar de à data da suspeição da FNC de Adilson Azul, a implantação de SRTN's em cada estado brasileiro já ter sido preconizada pelo PNTN, ${ }^{(10)}$ a equipe de saúde à qual o paciente teve acesso, encaminhou-o para SP, inviabilizando sua terapêutica.

A sintomatologia nos casos estudados não corresponderam aos achados de Steiner e cols (2007); (4) tampouco foram mais graves quando a ingestão proteica foi maior, apontando para manifestações fenotípicas distintas nos casos descritos.

Por tudo isso, considera-se que para além do tipo de mutações genéticas e alterações das taxas de fenilalanina, aspectos relacionados à dinâmica familiar e à aspectos subjetivos contribuíram para agravos nas manifestações clínicas da FNC - a in- 
tervenção interdisciplinar, considerando a estruturação psíquica dos sujeitos, deve ser preconizada.

Vê-se a importância da reavaliação da TN - questões práticas e subjetivas evidenciadas nas narrativas apontaram para a importância da ética do cuidado, em distintos setores a que se relacionaram: nas redes de apoio pública e privada, como suportes aos atendimentos dos pacientes; nas maternidades, quanto à adesão ao protocolo do PNTN; nos SRTNs, quanto à adoção de terapêutica e diretrizes conforme o PNTN.

O rastreio de casos tardios é imprescindível na promoção de casos sem tratamento.

Estudos que abordem a dimensão subjetiva precisam ser implementados visando melhor compreensão sobre distintos fenótipos na FNC.

\section{REFERÊNCIAS}

1. Freitag $\mathrm{CM}$. The genetics of autistic disorders and its clinical relevance: $A$ review of the literature. Mol. psychiatry. 2007;12:2-22.

2. Ghaziuddin M, Al-Owain M. Autism spectrum disorders and inborn errors of metabolism: an update. Pediatr. neurol. 2013;49(4):232-6.

3. Brasil. Ministério da Saúde; Portaria SAS/MS n 847, de 31 de outubro 2002. Institui o Protocolo clínico e diretrizes terapêuticas fenilcetonúria. Diário Oficial da União, Poder Executivo, Brasília, DF, O4 nov. 2002.

4. Steiner CE, Acosta AX, Guerreiro MM, Marquesde-Faria AP. Genótipo e história natural em indivíduos não aparentados com fenilcetonúria e comportamento autístico. Arq. neuro-psiquiatr. 2007;65(2A):2O2-5.

5. Lowe TL, Tanaka K, Seashore MR, Young JG, Cohen DJ. Detection of phenylketonuria in autistic and psychotic children. JAMA. 1980;243:126-8.

6. Chen $\mathrm{CH}$, Hsiao KJ. A Chinese classic phenylketonuria manifested as autism. Br. J. psychiatr. 1989;155:251-3.
7. Baieli S, Pavone L, Meli C, Fiumara A, Coleman M. Autism and Phenylketonuria. J. autism dev. disord. 2003;33(2):201-4.

8. Van Karnebeek CD, Stockler S. Treatable inborn errors of metabolism causing intellectual disability: a systematic literature review. Mol. genet. Metab. 2011;105:23)368-81.

9. Amorim T, Boa-Sorte N, Leite MEQ, Acosta, AX. Aspectos clínicos e demográficos da fenilcetonúria no Estado da Bahia. Rev. paul. pedatr. 2011;29(4):612-7.

10. Brasil. Ministério da Saúde. Portaria GM/MS n. ${ }^{\circ}$ 822/ GM, de 6 de junho de 2001. Instituição do Programa Nacional de Triagem Neonatal, no âmbito do Sistema Único de Saúde, para fenilcetonúria, hipotireoidismo congênito, fibrose cística e hemoglobinopatias. Brasília (DF); 2001.

11. Revista Autismo. Disponível em: http://www. revistaautismo.com.br

12. Pondé M, Rousseau C. Immigrant Children with Autism Spectrum Disorder: The Relationship between the Perspective of the Professionals and the Parents' Point of View. J. can. acad. child adolesc. psychiatry. 2013;22(2):131-8.

13. Battikha EC, Faria MCC de, Kopelman BI. As representações maternas acerca do bebê que nasce com doenças orgânicas graves. Psicol. teor. pesqui. 2007;23(1):17-24.

14. Jerusalinsky J. Temporalidade e Clínica com Bebês - uma abordagem da clínica da estimulação precoce a partir do corte epistemológico da psicanálise [dissertação]. São Paulo: Pontifícia Universidade Católica de São Paulo, 2003.

15. Vorcaro A, Lucero A. A criança e a debilidade mental: uma abordagem lacaniana. Psicol. USP, 2011;22(4):813-832.

16. Wallon H. Criança turbulenta: estudo sobre os retardamentos e as anomalias do desenvolvimento motor e mental. Rio de Janeiro: Vozes; 2007.

17. Levin E. A clínica psicomotora: o corpo na linguagem. Petrópolis, RJ: Vozes; 1995.

18. Laznik MC. A voz da sereia: o autismo e os impasses na constituição do sujeito. Salvador: Ágalma; 2004. p. 27-31. 
19. Frizzo GB, Piccinini CA. Interação mãebebê em contexto de depressão materna: aspectos teóricos e empíricos. Psicol. estud. 2005;10(1):47-55.

20. Wallack L, Winkleby M. Primary prevention: A new look at basic concepts. Soc. sci. med. 1987;25(8):923-30.

21. Berlinguer G. Bioética da prevenção. Bioética. 1994;2(2):117-22.

22. Minayo MCDS. O desafio do conhecimento: pesquisa qualitativa em saúde. São Paulo: Hucitec; 2010.

23. Valles MS. Entrevistas cualitativas. Madrid: Centro de Investigaciones Sociológicas; 2002.
24. Gil AC. Como elaborar projetos de pesquisa. $4^{a}$ ed. São Paulo: Atlas; 2002.

25. Amorim T, Abé-Sandes K, Castilla E, Grossi G, Vieira T, Queiroz I et al. Genetics in the "Sertão": Study of frequent monogenic disorders in Monte Santo - a small city of the state of Bahia - northeastern Brazil. Acta bioq. Latinoamer. 2007;194(supl 1).

26. Amorim T. Estudo das bases moleculares da fenilcetonúria no nordeste do Brasil [tese]. Salvador: Fundação Oswaldo Cruz, Centro de Pesquisas Gonçalo Moniz, 2010.

27. Teixeira LC. Função paterna, frátria e violência: sobre a constituição do socius na psicanálise freudiana. Psico-USF. 2002;7(2):195-200. 